\title{
Synchrotron Radiation Photoemission Spectroscopy for Native Oxide Layer on Vanadium and VCrTa
}

\author{
Yuden Teraoka, Akitaka Yoshigoe, James Harries \\ Japan Atomic Energy Agency, 1-1-1 Kouto, Sayo-cho, Sayo-gun, Hyogo 679-5148, Japan \\ yteraoka@spring8.or.jp
}

(Received : December 29, 2008; Accepted: February 10, 2009)

\begin{abstract}
In order to study correlation between oxide layers on hydrogen storage metals such as V(111), polycrystalline Vanadium, and VCrTa alloy and hydrogen desorption temperature, the surfaces covered by native oxides have been analyzed by photoemission spectroscopy using soft $\mathrm{x}$-ray synchrotron radiation. Depth analyses of the oxide layers were performed by changing synchrotron radiation energy to estimate the oxide thickness. The native oxide layer was disappeared by thermal annealing up to $713 \mathrm{~K}$ for the $\mathrm{V}(111)$ surface. The oxide layers of the poly $\mathrm{V}$ and the VCrTa alloy, however, were remained even at $873 \mathrm{~K}$. Although the oxides of $\mathrm{V}$ and $\mathrm{Cr}$ were disappeared, Ta oxides were remained. The re-arrangement of oxidation took place in the oxide layer of the $\mathrm{VCrTa}$ alloy by thermal annealing. The oxidation of Ta controlled oxide thickness in the VCrTa alloy. The formation of an artificial oxide layer could be controlled using supersonic oxygen molecular beams.
\end{abstract}

\section{Introduction}

Hydrogen storage materials such as Vanadium compounds are important as candidates for fuel cells of automobiles [1]. In generally speaking, desorption and absorption of hydrogen molecules in the hydrogen storage materials take place through native oxide layers formed on the surface. Thus, it is important to study the nature of the oxide layer to control thermal desorption characteristics of hydrogen molecules. In order to study correlation between hydrogen desorption temperature and chemical bonding states of the oxide layer, photoemission spectroscopy with high brilliance and high energy-resolution synchrotron radiation (SR-XPS) has been applied for the analyses of the native oxide, its thermal instability, and the reformation of artificial oxides at the surfaces of single crystalline $\mathrm{V}(111)$, polycrystalline Vanadium and polycrystalline VCrTa alloy.

\section{Experimental}

All experiments have been performed at the surface chemistry experimental station (SUREAC2000) of the BL23SU in the SPring-8 facilities [2]. The base vacuum pressure in this apparatus was less than $2 \times 10^{-8} \mathrm{~Pa}$. Photoelectrons were detected by a concentric hemispherical analyzer with $5 \mathrm{MCD}$. Photon energy used in this work was tuned from $592 \mathrm{eV}$ to $1569 \mathrm{eV}$. The SR beam of $1247 \mathrm{eV}$ was used for thermal instability and re-oxidation studies. The detection angle of photoelectrons was 30 degrees with respect to surface normal. Photoemission spectra for O-1s, C-1s, V-2p, V-3p, Cr-2p, Ta-4d and a wide scan were measured. Binding energy values of the inner shells were referred from a data book [3].

The thermal radiation from a Ta ribbon heater, which was set in the backside of sample, was used for sample heating. The thermal instability of native oxide layers was observed by the SR-XPS at room temperature after heating up to the destination temperature to avoid effects of a magnetic field generated in the Ta ribbon heater.

The supersonic $\mathrm{O}_{2}$ molecular beams (SSOMB) were used for the artificial oxides formation. Translational kinetic energy of the SSOMB is controllable from $0.02 \mathrm{eV}$ to $2.3 \mathrm{eV}$ by changing nozzle temperature and gas mixing ratio $\left(\mathrm{O}_{2} / \mathrm{He} / \mathrm{Ar}\right)$. The energy value was calculated on the basis of the energy conservation law before and after adiabatic gas expansion from a nozzle orifice. After an appropriate $\mathrm{O}_{2}$ beam dosage, photoelectron spectra were measured. The dosage and XPS measument cycle was continued until oxygen saturation. The $\mathrm{O}_{2}$ flux density of the SSOMB was estimated from measurements of gas pressure increase in the reaction chamber, the gas mixing ratio, and the molecular beam spot size at the sample surface.

\section{Results and Discussion}

\subsection{Analyses of Native Oxide by Changing Synchrotron Radiation Energy}

Photoemission spectra of V-2p and O-1s for the native oxide layer on the $\mathrm{V}(111)$ surface is shown in Fig. 1. A synchrotron radiation energy dependence 
was measured to estimate thickness of the layer. $\mathrm{V}-2 \mathrm{p}$ photoemission peaks contributed from bulk could be observed with photon energy larger than $795 \mathrm{eV}$ so that the thickness might be about $2 \mathrm{~nm}$ assuming the detectable thickness was three times larger than escape depth. The O-1s photoemission spectrum consisted of at least two components. A shoulder structure seen in higher binding energy was changed to be minor in higher SR energy so that the component was surface sensitive.

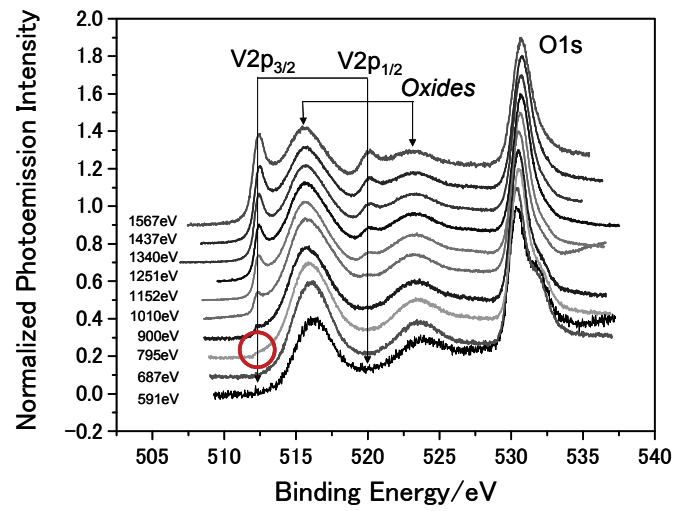

Fig. 1 Synchrotron radiation energy dependence of V-2p and $\mathrm{O}-1 \mathrm{~s}$ peaks for a native oxide layer on $\mathrm{V}(111)$.

A synchrotron radiation energy dependence of $\mathrm{V}-2 \mathrm{p}$ and $\mathrm{O}-1 \mathrm{~s}$ photoemission spectra for the poly $\mathrm{V}$ surface is shown in Fig. 2. The profile is almost same as Fig. 1. V-2p peaks from the bulk could be observed from $795 \mathrm{eV}$ so that the oxide thickness was estimated to be about $2 \mathrm{~nm}$. The O-1s peak profile was also resembled with Fig. 1.

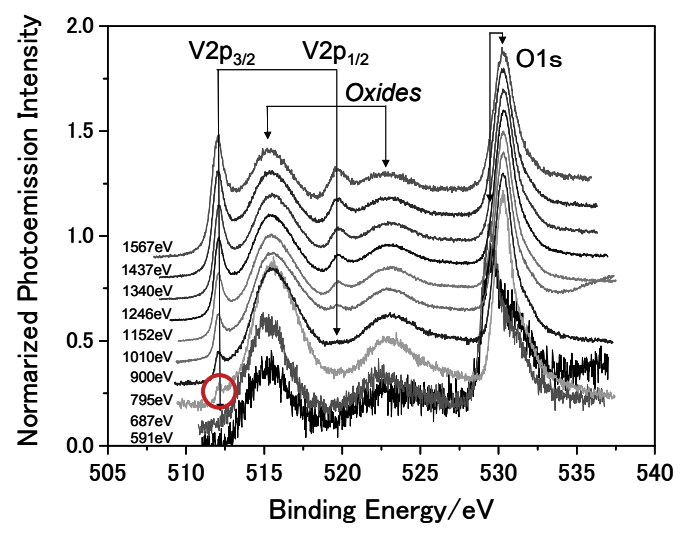

Fig. 2 Synchrotron radiation energy dependence of V-2p and $\mathrm{O}-1 \mathrm{~s}$ peaks for a native oxide layer on poly $\mathrm{V}$.

A synchrotron radiation energy dependence of $\mathrm{V}-2 \mathrm{p}$ and $\mathrm{O}-1 \mathrm{~s}$ peaks for the VCrTa alloy is shown in Fig. 3. In contrast to the former two cases, the $\mathrm{O}-1$ s peak and chemically-shifted $\mathrm{V}-2 \mathrm{p}$ peaks are larger than those in Figs. 1 and 2. It implies that further oxidation proceeds in this alloy comparing with the V(111) and the poly V. Indeed, V-2p peaks contributed from the bulk could be observed with photon energy larger than $1012 \mathrm{eV}$ so that the oxide thickness might be about $2.4 \mathrm{~nm}$. Although the $\mathrm{O}-1 \mathrm{~s}$ peak profile showed an asymmetric shape tailing to the higher binding energy side, the surface sensitive component was not clearly observed.

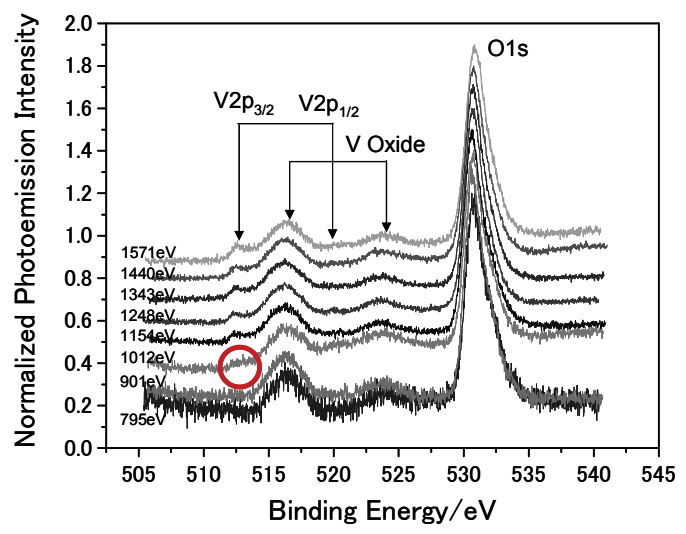

Fig. 3 Synchrotron radiation energy dependence of V-2p and $\mathrm{O}-1 \mathrm{~s}$ peaks for a native oxide layer on $\mathrm{VCrTa}$ alloy.

It is interesting to investigate oxidation states of $\mathrm{Cr}$ and $\mathrm{Ta}$ to consider the origin of thicker oxide layer formation. Synchrotron radiation energy dependences of $\mathrm{Cr}-2 \mathrm{p}$ and Ta- $4 \mathrm{~d}$ peaks are shown in Figs. 4 and 5. Cr-2p peaks contributed from bulk could be observed with photon energy larger than $1012 \mathrm{eV}$ as well as V-2p. Ta-4d peaks, however, could be observed from $1440 \mathrm{eV}$ so that the oxide thickness might be about $4 \mathrm{~nm}$. These deeper oxygen atoms might be the origin of very small SR energy dependence of O-1s peak.

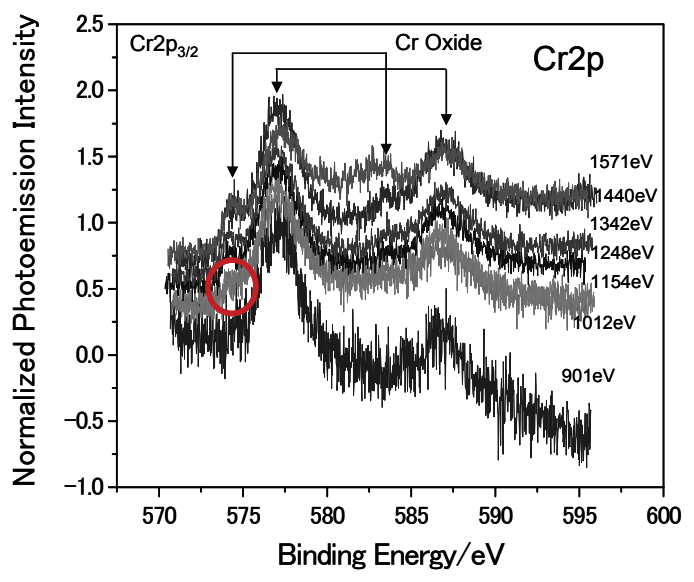

Fig. 4 Synchrotron radiation energy dependence of $\mathrm{Cr}-2 \mathrm{p}$ peaks for a native oxide layer on VCrTa alloy. 


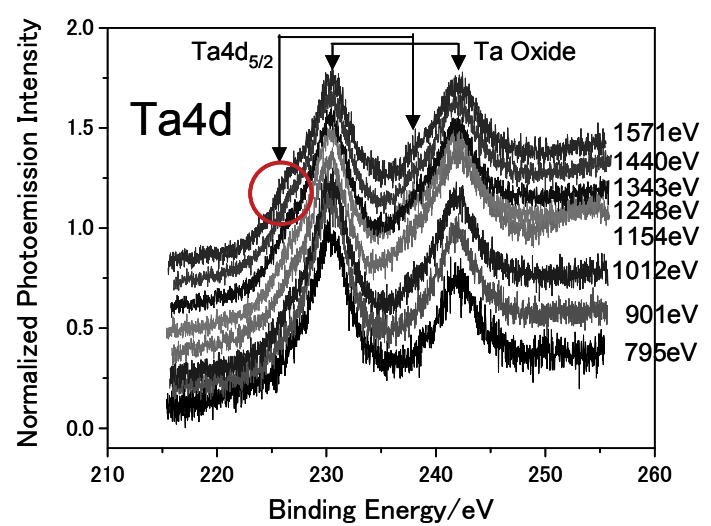

Fig. 5 Synchrotron radiation energy dependence of Ta-4d peaks for a native oxide layer on VCrTa alloy.

\subsection{Thermal Instability of Native Oxide}

Thermal instability of the native oxide layer has been investigated by flash heating. Fig. 6 shows $\mathrm{V}-2 \mathrm{p}$ and $\mathrm{O}-1 \mathrm{~s}$ variation depending on thermal annealing temperature ranging from $713 \mathrm{~K}$ and $1513 \mathrm{~K}$ for the $\mathrm{V}(111)$ surface. The bulk components of $\mathrm{V}-2 \mathrm{p}_{1 / 2}$ and $\mathrm{V}-2 \mathrm{p}_{3 / 2}$ peaks increased with decreasing O-1s peak. The O-1s peak decreased to be negligible even at $713 \mathrm{~K}$. Small doublet peaks, however, remained even after heating up to $1513 \mathrm{~K}$. The higher binding energy component is surface sensitive and the other peak corresponds to the main native oxide peak.

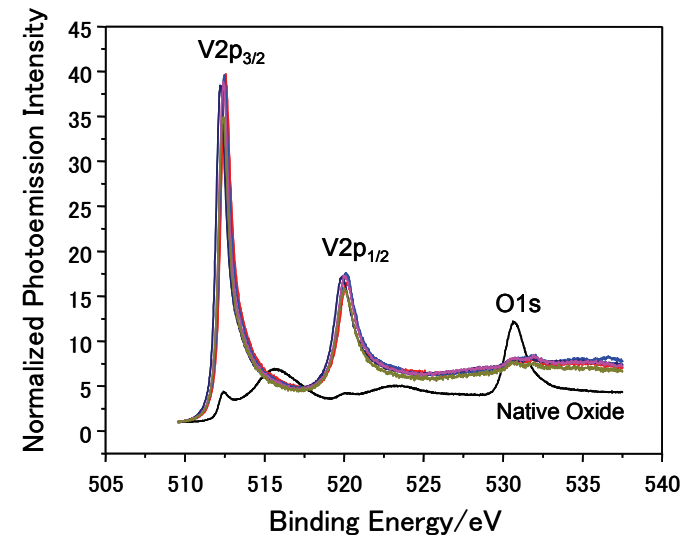

Fig. 6 Thermal annealing effects for V-2p and O-1s peaks of a native oxide layer on $\mathrm{V}(111)$ by heating at 713 $\mathrm{K}$ to $1513 \mathrm{~K}$ observed with the photon energy of $1247 \mathrm{eV}$

The similar experiments were performed for the poly V. The results were indicated in Fig. 7. Although all photoemission spectra were not changed up to $573 \mathrm{~K}$, the O-1s peak decreased and the bulk components of $\mathrm{V}-2 \mathrm{p}$ peaks increased at 673 $\mathrm{K}$. The $\mathrm{V}-2 \mathrm{p}_{3 / 2}$ peak position was shifted slightly and a satellite peak structure was observed as well due to high energy-resolution SR-XPS as shown in Fig. 8. The peak shift may be attributed to a structure reconstruction at the surface by surface melting. And the satellite structure may correspond to sub-oxides because oxygen coverage decreased with increasing temperature. The O-1s peak disappeared completely at $1273 \mathrm{~K}$. A clean surface could be obtained only by thermal annealing in the ultra high vacuum condition.

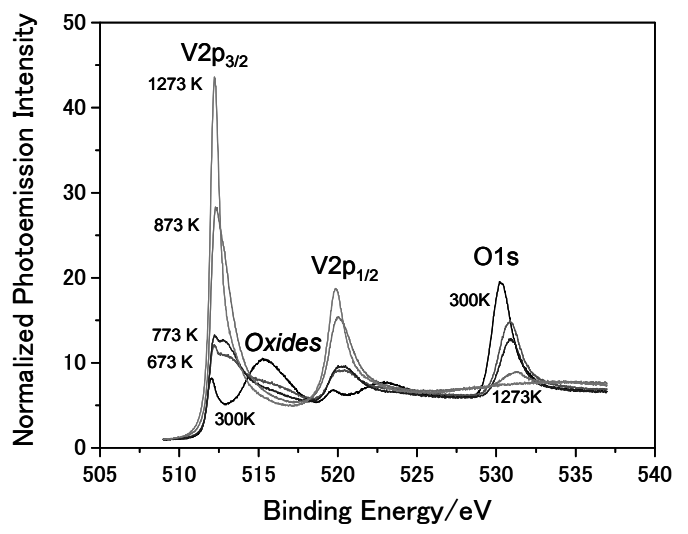

Fig. 7 Thermal annealing effects for $\mathrm{V}-2 \mathrm{p}$ and $\mathrm{O}-1$ s peaks of a native oxide layer on poly $\mathrm{V}$ observed with the photon energy of $1247 \mathrm{eV}$.

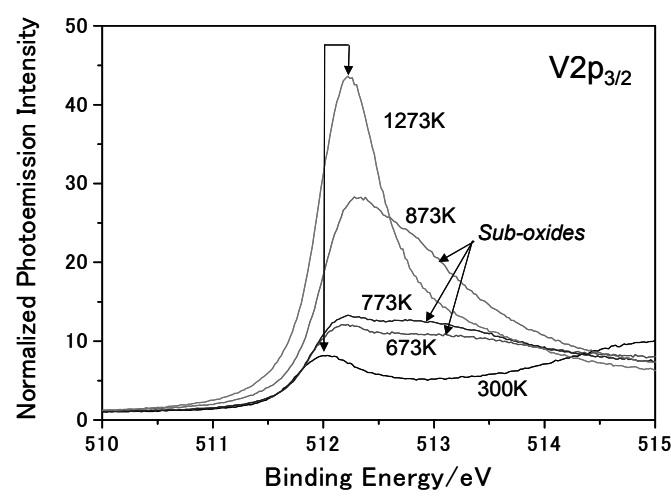

Fig. 8 Thermal annealing effects for $V-2 p_{3 / 2}$ peak of a native oxide layer on poly $\mathrm{V}$ observed with the photon energy of $1247 \mathrm{eV}$.

In contrast to the single element $\mathrm{V}$, thermal instability of the native oxide layer of the $\mathrm{VCrTa}$ alloy was complicated. Although $\mathrm{V}$ peaks from bulk grew with increasing temperature as seen in the single element $\mathrm{V}$ cases, the $\mathrm{O}-1 \mathrm{~s}$ peak was remained as shown in Fig. 9.

Thermal annealing effects for $\mathrm{Cr}-2 \mathrm{p}$ peaks are shown in Fig. 10. Chemically-shifted oxide peaks were disappeared and bulk components grew to be main peaks as seen in the V-2p case. These results indicate that $\mathrm{Cr}-\mathrm{O}$ bonds are broken and metal $\mathrm{Cr}$ or its alloy is segregated on the surface. On the other hand, chemically-shifted components of Ta-4d 
peaks were not disappeared by heating as shown in Fig.11. Ta-O bonds are still remained even up to $873 \mathrm{~K}$. These facts imply that oxygen atoms bonding with $\mathrm{V}$ and $\mathrm{Cr}$ may be transferred to Ta.

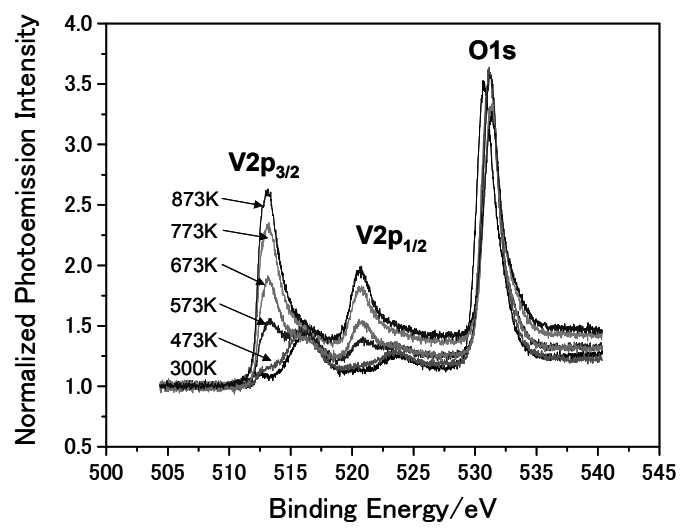

Fig. 9 Thermal annealing effects for $\mathrm{V}-2 \mathrm{p}$ and $\mathrm{O}-1 \mathrm{~s}$ peaks of a native oxide layer on $\mathrm{VCrTa}$ alloy observed with the photon energy of $1247 \mathrm{eV}$.

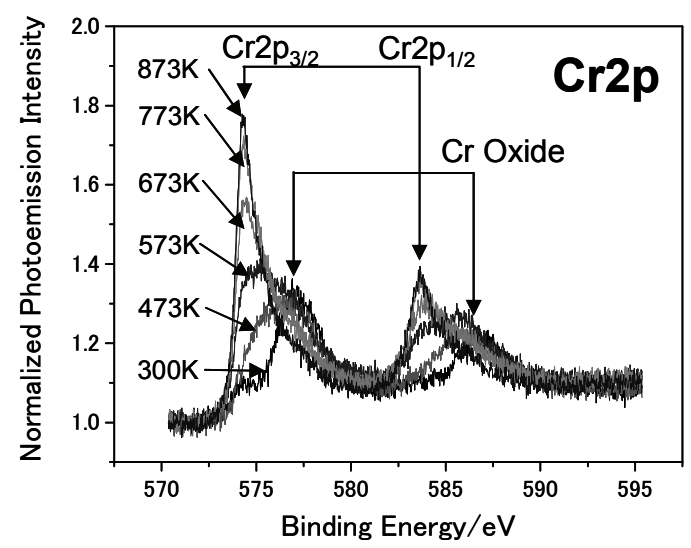

Fig. 10 Thermal annealing effects for $\mathrm{Cr}-2 \mathrm{p}$ peaks of a native oxide layer on $\mathrm{VCrTa}$ alloy observed with the photon energy of $1247 \mathrm{eV}$.

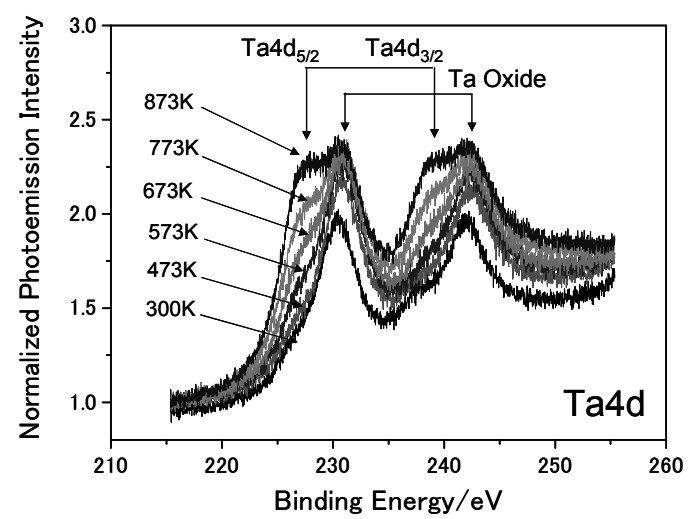

Fig. 11 Thermal annealing effects for Ta-4d peaks of a native oxide layer on $\mathrm{VCrTa}$ alloy observed with the photon energy of $1247 \mathrm{eV}$.

\subsection{Artificial Oxide Formation at V(111)}

The clean surfaces of $\mathrm{V}(111)$ and poly $\mathrm{V}$, formed by heating up to $1273 \mathrm{~K}$, were irradiated by SSOMB with $2.3 \mathrm{eV}$ until oxygen saturation after cooling down to room temperature. In both cases, similar oxide layers were formed. The spectral change of $\mathrm{V}-2 \mathrm{p}$ and $\mathrm{O}-1 \mathrm{~s}$ for the $\mathrm{V}(111)$ surface is shown in Fig. 12. The bulk V-2p peak intensity was larger than that for the native oxide layer. The bulk sensitive component of O-1s peak grew to be major. Although the reason why the differences of $V-2 p$ and its oxide peaks between the native oxide and the artificial oxide was now under investigation, it was confirmed that the oxidation could be controlled by SSOMB in the present preliminary experiments.

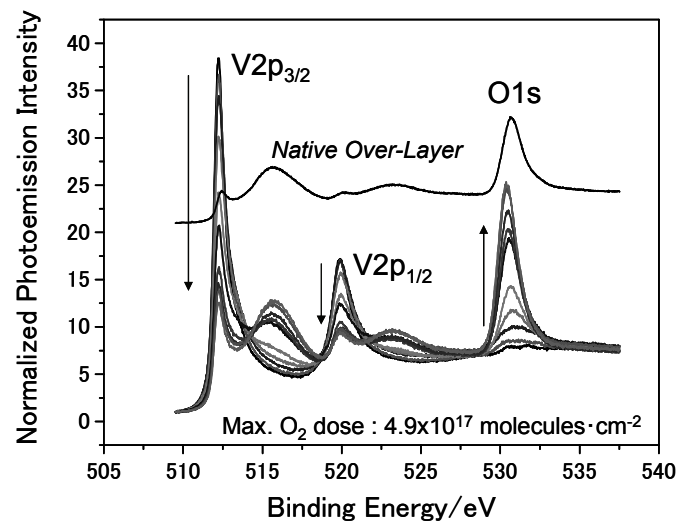

Fig. 12 Artificial oxide formation at V(111) surface by irradiation of supersonic oxygen molecular beams with translational energy of $2.3 \mathrm{eV}$. The $\mathrm{V}-2 \mathrm{p}$ peaks decreased and in turn $\mathrm{O}-1 \mathrm{~s}$ peak increased during $\mathrm{O}_{2}$ beam irradiation up to $4.9 \times 10^{17}$ molecules $\cdot \mathrm{cm}^{-2}$ until saturation. The photon energy was $1247 \mathrm{eV}$.

\section{Acknowledgements}

This work has been supported by New Energy and Industrial Technology Development Organization (NEDO) under "Advanced Fundamental Research Project on Hydrogen Storage Materials".

\section{References}

[1] H. Arishima, F. Takahashi, T. Ebisawa, H. Itoh and T. Kabutomori, J. Alloy and Comp. 405, 356 (2003).

[2] Y. Teraoka, A. Yoshigoe, and K. Moritani, Electrical Engineering in Japan 164(3), 60 (2008).

[3] J. Emsley, The Elements, 3rd Edn., Oxford University Press, Oxford (1998). 